\title{
Blow-up time estimates in nonlocal reaction-diffusion systems under various boundary conditions
}

Monica Marras ${ }^{*}$ (D) and Stella Vernier Piro

${ }^{*}$ Correspondence:

mmarras@unica.it

Dipartimento di Matematica e

Informatica, Universitá di Cagliari, V.

le Merello 92, Cagliari, 09123, Italy

\begin{abstract}
This paper deals with the question of blow-up of solutions to nonlocal reaction-diffusion systems under various boundary conditions. Specifically, conditions on data are introduced to avoid the blow-up of the solution and, when the blow-up occurs, explicit lower and upper bounds of blow-up time are derived.
\end{abstract}

MSC: 35B44; 35A01; 35K51

Keywords: blow-up; global existence; nonlinear parabolic systems

\section{Introduction}

The purpose of this paper is to study the blow-up phenomenon of nonnegative solutions for some classes of reaction-diffusion systems under different boundary conditions and when the reaction terms have a nonlocal functional dependence in space- and timedependent coefficients.

Let us consider the following system:

$$
\left\{\begin{array}{l}
u_{t}=\Delta u+k_{1}(t) u^{p} \int_{\Omega} v^{q} d x \quad \text { in } \Omega \times\left(0, t^{*}\right), \\
v_{t}=\Delta v+k_{2}(t) v^{p} \int_{\Omega} u^{q} d x \quad \text { in } \Omega \times\left(0, t^{*}\right), \\
\beta \frac{\partial u}{\partial v}+\alpha u=0 \quad \text { on } \partial \Omega \times\left(0, t^{*}\right), \\
\beta \frac{\partial v}{\partial v}+\alpha v=0 \quad \text { on } \partial \Omega \times\left(0, t^{*}\right), \\
u(x, 0)=u_{0}(x) \quad \text { on } \Omega, \\
v(x, 0)=v_{0}(x) \quad \text { on } \Omega,
\end{array}\right.
$$

where the spatial domain $\Omega \subset \mathbb{R}^{N}$ is bounded with smooth boundary $\partial \Omega, t^{*}$ is the blowup time, $k_{1}, k_{2}$ are two positive functions of $t$, and $\alpha, \beta \geq 0$. We assume that the initial data $u_{0}(x), v_{0}(x)$ are nonnegative functions satisfying the compatibility condition on $\partial \Omega$; then by the maximum principle [1] the solution of (1.1) is nonnegative in its time interval of existence $[0, \tau], \tau<t^{*}$.

The two equations in (1.1) are completely coupled via the nonlocal nonlinear sources with $p, q>0$.

(c) The Author(s) 2017. This article is distributed under the terms of the Creative Commons Attribution 4.0 International License (http://creativecommons.org/licenses/by/4.0/), which permits unrestricted use, distribution, and reproduction in any medium, provided you give appropriate credit to the original author(s) and the source, provide a link to the Creative Commons license, and indicate if changes were made. 
There are some important phenomena formulated as parabolic equations that are coupled with nonlocal boundary conditions in mathematical modeling such as thermoelasticity theory (see [2, 3], and [4]). In this case, the solution can be used to describe the entropy per volume of the material. We remark that nonlocal terms may appear also on the boundary conditions. Friedman [4] investigated the behavior of the solutions of the system

$$
\left\{\begin{array}{l}
u_{t}-A u=0, \quad x \in \Omega, t>0 \\
u(x, t)=\int_{\Omega} f(x, y) u(y, t) d y, \quad x \in \partial \Omega, t>0 \\
u(x, 0)=u_{0}(x), \quad x \in \bar{\Omega}
\end{array}\right.
$$

where $A$ is a uniformly elliptic operator.

As for more general discussions on the dynamics of parabolic problems with nonlocal boundary conditions, we refer to Pao [5], where the following problem was considered:

$$
\left\{\begin{array}{l}
u_{t}-A u=h(x, u), \quad x \in \Omega, t>0 \\
\alpha_{0} \frac{\partial u}{\partial v}+u=\int_{\Omega} f(x, y) u(y, t) d y, \quad x \in \partial \Omega, t>0 \\
u(x, 0)=u_{0}(x), \quad x \in \bar{\Omega}
\end{array}\right.
$$

Recently, Kong and Wang [6] obtained the blow-up conditions and blow-up profiles of the following system by using some ideas of Souplet [7]:

$$
\left\{\begin{array}{l}
u_{t}=\Delta u+\int_{\Omega} u^{\alpha} v^{p} d x, \quad v_{t}=\Delta v+\int_{\Omega} u^{q} v^{\beta} d x, \quad x \in \Omega, t>0, \\
u(x, t)=\int_{\Omega} f(x, y) u(x, y) d y, \quad v(x, t)=\int_{\Omega} g(x, y) v(x, y) d y, \quad x \in \partial \Omega, t>0, \\
u(x, 0)=u_{0}(x), \quad v(x, 0)=v_{0}(x), \quad x \in \bar{\Omega} .
\end{array}\right.
$$

Furthermore, Zheng and Kong [8] gave conditions for the global existence or nonexistence of a solution to the following system:

$$
\left\{\begin{array}{l}
u_{t}=\Delta u+u^{\alpha} \int_{\Omega} v^{p} d x, \quad v_{t}=\Delta v+v^{\beta} \int_{\Omega} u^{q} d x, \quad x \in \Omega, t>0, \\
u(x, t)=\int_{\Omega} f(x, y) u(x, y) d y, \quad v(x, t)=\int_{\Omega} g(x, y) v(x, y) d y, \quad x \in \partial \Omega, t>0, \\
u(x, 0)=u_{0}(x), \quad v(x, 0)=v_{0}(x), \quad x \in \bar{\Omega} .
\end{array}\right.
$$

Souplet [9] studied the blow-up behavior of nonnegative solutions for some classes of reaction-diffusion equations, where the reaction term may have a nonlocal functional dependence either in space or in time (or possibly in both space and time). For each type of problems, the author gave finite time blow-up results that significantly improved or extended previous results of several authors.

Marras and Vernier Piro [10] considered the following class of reaction-diffusion systems subject to nonlocal boundary conditions:

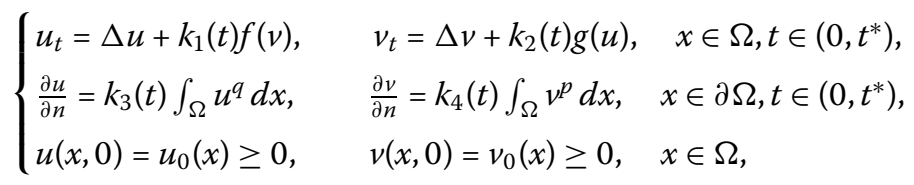


where $\Omega$ is a bounded convex domain in $\mathbb{R}^{N}, N \geq 2$, with smooth boundary, and $f, g, u_{0}$, and $v_{0}$ are smooth nonnegative functions. The authors prove that, under certain conditions on the data, the blow-up occurs at some finite time $t^{*}$, and, when its does, they derive explicit lower and upper bounds. The case of a single equation is analyzed in [11].

If the source term is local, for instance, of power type, results on blow-up behavior of the solutions to parabolic problems under Dirichlet, Neumann, and Robin boundary conditions are present in [12] and [13] (see also [14]). In the case of a source term combination of a nonlocal term with an exponential one, Pao points out applications to thermal explosion in combustion theory (see [15] and the references therein).

The novelty of this paper is in associating with system (1.1) Dirichlet, Neumann, and Robin boundary conditions and to present methods working in all the cases with the aim to obtain upper and lower bounds for blow-up time and also to prove the global existence of solutions. Nevertheless, we treat separately the three cases since the proofs in the Dirichlet problem $(\beta=0)$ and in the Neumann problem $(\alpha=0)$ are not particular cases of the Robin problem: in fact, we use the properties connected with the eigenvalues of the fixed, free, and elastically supported membrane problems, respectively, defined in problems (2.6), (3.3), and (4.3) (see [16]).

For other interesting results concerning the membrane response, which includes an elastic response and viscous behavior, the readers may refer to $[17,18]$.

The paper is organized as follows. In Section 2 (Section 2.1), we consider a spatial domain $\Omega \subset \mathbb{R}^{N}$ and derive an upper bound of the blow-up time by constructing a blowing up subsolution of our problem, which implies that our solution also blows up. In Sections 2.2 and 2.3, we restrict our investigation to a domain $\Omega \subset \mathbb{R}^{3}$ and obtain respectively a lower bound for $t^{*}$ and the conditions to avoid the blow-up phenomenon.

In Section 3, we extend results in Section 2 to our problem when the Dirichlet boundary condition is replaced by the Neumann one. In particular, in Sections 3.1 and 3.2, the extension is immediate; however, in Section 3.3, we have to rely on an inequality that allows us to estimate the integral term containing the gradient of the solution.

In Section 4, under appropriate variations, the results of Section 2 are extended. Specifically, in order to obtain a lower bound of $t^{*}$ and the nonblow-up of the solution, to manage the boundary integral term, we use the variational definition of the first eigenvalue of the elastically supported problem.

Throughout the paper, for clarity, we indicate with $t_{\mathcal{D}}^{*}, t_{\mathcal{N}}^{*}$, and $t_{\mathcal{R}}^{*}$ the blow-up times of the solutions to (1.1) under Dirichlet, Neumann, and Robin boundary conditions, respectively.

\section{Estimates of $t_{\mathcal{D}}^{*}$}

In this section, we consider system (1.1) under the Dirichlet boundary condition ( $\beta=0$ and $\alpha=1$ ):

$$
\left\{\begin{array}{l}
u_{t}=\Delta u+k_{1}(t) u^{p} \int_{\Omega} v^{q} d x \quad \text { in } \Omega \times\left(0, t_{\mathcal{D}}^{*}\right), \\
v_{t}=\Delta v+k_{2}(t) v^{p} \int_{\Omega} u^{q} d x \quad \text { in } \Omega \times\left(0, t_{\mathcal{D}}^{*}\right), \\
u=0, \quad v=0 \quad \text { on } \partial \Omega \times\left(0, t_{\mathcal{D}}^{*}\right), \\
u(x, 0)=u_{0}(x) \geq 0, \quad v(x, 0)=v_{0}(x) \geq 0 \quad \text { on } \Omega .
\end{array}\right.
$$




\subsection{Upper bound for $t_{\mathcal{D}}^{*}$}

We will prove that the solution $(u, v)$ blows up in finite time $t_{\mathcal{D}}^{*}$ and derive an upper bound of $t_{\mathcal{D}}^{*}$. To this end, we construct a blowing up subsolution $(\underline{u}, \underline{v})$ of $(2.1)$.

We recall that $(\underline{u}, \underline{v}) \in C^{2,1}(Q) \cup C(\bar{Q}), Q=\Omega \times(0, T)$, is a subsolution of (2.1) if

$$
\left\{\begin{array}{l}
\underline{u}_{t}-\Delta \underline{u}-k_{1}(t) \underline{u}^{p} \int_{\Omega} \underline{v}^{q} d x \leq 0 \quad \text { in } \Omega \times(0, T), \\
\underline{v}_{t}-\Delta \underline{v}-k_{2}(t) \underline{v}^{p} \int_{\Omega} \underline{u}^{q} d x \leq 0 \quad \text { in } \Omega \times(0, T), \\
\underline{u} \leq 0, \quad \underline{v} \leq 0 \quad \text { on } \partial \Omega \times(0, T), \\
\underline{u}(x, 0) \leq u_{0}, \quad \underline{v}(x, 0) \leq v_{0} \quad \text { on } \Omega,
\end{array}\right.
$$

so that if $(\underline{u}, \underline{v})$ blows up at time $T$, that is,

$$
\lim _{t \rightarrow T}(\underline{u}, \underline{v})=+\infty,
$$

then $(u, v)$ blows up in a finite time $t_{\mathcal{D}}^{*}<T$.

In order to find a subsolution of (2.1), we first prove the following:

Lemma 2.1 Let $s(t)$ be the unique solution of the problem

$$
\left\{\begin{array}{l}
s^{\prime}(t)=-a_{1} s(t)+a s^{\gamma}(t), \quad a_{1}, a>0, \gamma>1, \\
s(0)=s_{0}
\end{array}\right.
$$

with constant

$$
s_{0}>\left(\frac{a_{1}}{a}\right)^{\frac{1}{\gamma-1}} .
$$

Then $s(t)$ blows up in finite time

$$
T=\ln \left[\left(\frac{a s_{0}^{\gamma-1}}{a s_{0}^{\gamma-1}-a_{1}}\right)^{\frac{1}{(\gamma-1) a_{1}}}\right] .
$$

Proof We easily find the solution of (2.3):

$$
s(t)=\left[\frac{a}{a_{1}}-\left(\frac{a s_{0}^{\gamma-1}-a_{1}}{a_{1} s_{0}^{\gamma-1}}\right) e^{(\gamma-1) a_{1} t}\right]^{-\frac{1}{\gamma-1}},
$$

which blows up at time $T$ defined in (2.4).

Let $\Omega$ be a bounded domain in $\mathbb{R}^{N}$, and let us denote

$$
\left\{\begin{array}{l}
\gamma=\min \{m(p-1)+n q+1, n(p-1)+m q+1\}>1, \\
k=\min _{(0, T)}\left\{k_{1}(t), k_{2}(t)\right\}, \\
a_{1}=2 \lambda_{1}, \\
a=\min _{x \in \Omega}\left\{\frac{1}{m} k \varphi_{1}^{2 m(p-1)}|\Omega|^{1-m q}, \frac{1}{n} k \varphi_{1}^{2 n(p-1)}|\Omega|^{1-n q}\right\}
\end{array}\right.
$$


where $|\Omega|$ is the measure of $\Omega, m, n \geq 1, p>1, q>1$, and $\varphi_{1}$ and $\lambda_{1}$ are respectively the first eigenfunction and the corresponding eigenvalue of the fixed membrane problem

$$
\left\{\begin{array}{l}
\Delta \varphi(x)+\lambda \varphi(x)=0, \quad \varphi(x)>0, x \in \Omega, \\
\varphi(x)=0, \quad x \in \partial \Omega,
\end{array}\right.
$$

with

$$
\int_{\Omega} \varphi_{1}^{2}(x) d x=1
$$

We seek an unbounded subsolution of (2.1) of the form

$$
\left\{\begin{array}{l}
\underline{u}:=s(t)^{n} \varphi_{1}(x)^{2 n} \\
\underline{v}:=s(t)^{m} \varphi_{1}(x)^{2 m}
\end{array}\right.
$$

with $\varphi_{1}$ the first eigenfunction of (2.6), $m, n \geq 1$, and $s(t) \in C^{1}$ the solution of (2.3). We note that $(\underline{u}, \underline{v})$ blows up in finite time $T$. We now prove that $(\underline{u}, \underline{v})$ is a subsolution of (2.1).

Theorem 2.1 Let $(u, v)$ be the solution of (2.1). Assume that Lemma 2.1 holds. If

$$
u_{0} \geq s_{0}^{n} \varphi_{1}^{2 n}, \quad v_{0} \geq s_{0}^{m} \varphi_{1}^{2 m}, \quad m, n \geq 1,
$$

then $(u, v)$ blows up in finite time $t^{*}$, and

$$
t_{\mathcal{D}}^{*} \leq T=\ln \left[\left(\frac{a s_{0}^{\gamma-1}}{a s_{0}^{\gamma-1}-2 \lambda_{1}}\right)^{\frac{1}{2(\gamma-1) \lambda_{1}}}\right] .
$$

Proof We consider $(\underline{u}, \underline{v})$ defined in (2.8) and compute

$$
\begin{aligned}
\underline{u}_{t} & -\Delta \underline{u}-k_{1}(t) \underline{u}^{p} \int_{\Omega} \underline{v}^{q} d x \\
& =n s^{n-1} s^{\prime} \varphi^{2 n}-2 n(2 n-1) s^{n} \varphi_{1}^{2(n-1)}|\nabla \varphi|^{2}+2 n \lambda_{1} s^{n} \varphi_{1}^{2 n}-k_{1}(t) s^{n p+m q} \varphi_{1}^{2 n p} \int_{\Omega} \varphi_{1}^{2 m q} d x \\
& \leq n s^{n-1} s^{\prime} \varphi^{2 n}+2 n \lambda_{1} s^{n} \varphi_{1}^{2 n}-k|\Omega|^{1-m q} s^{n p+m q} \varphi_{1}^{2 n p} \\
& =n s^{n-1} \varphi_{1}^{n}\left[s^{\prime}+2 \lambda_{1} s-\frac{k|\Omega|^{1-m q}}{n} s^{n(p-1)+m q+1} \varphi_{1}^{2 n(p-1)}\right] .
\end{aligned}
$$

In the last step, we have used the Hölder inequality and definition (2.5) of $k$ and (2.7). Since $\gamma>1$ and $s(t)$ is the solution of (2.3) that blows up at time $T$, taking into account (2.5), inequality (2.11) becomes

$$
\begin{aligned}
\underline{u}_{t} & -\Delta \underline{u}-k_{1}(t) \underline{u^{p}} \int_{\Omega} \underline{v}^{q} d x \\
& \leq n s^{n-1} \varphi_{1}^{2 n}\left(a s^{\gamma}-\frac{k|\Omega|^{1-m q}}{n} s^{n(p-1)+m q+1} \varphi_{1}^{2 n(p-1)}\right) \leq 0 .
\end{aligned}
$$


Moreover,

$$
\underline{u}(x, t)=s(t)^{n} \varphi_{1}(x)^{2 n}=0 \quad \text { in } \partial \Omega \times(0, T),
$$

and initially

$$
\underline{u}(x, 0)=s_{0}^{n} \varphi_{1}(x)^{2 n} \leq u_{0}(x) \text { in } \Omega .
$$

Then $\underline{u}(x, t) \leq u(x, t)$.

Similarly,

$$
\left\{\begin{array}{l}
\underline{v}_{t}-\Delta \underline{v}-k_{2}(t) v^{p} \int_{\Omega} \underline{u}^{q} d x \leq 0 \quad \text { in } \Omega \times(0, T) \\
\underline{v}=0 \quad \text { in } \partial \Omega \times(0, T), \\
\underline{v}(x, 0)=s_{0}^{m} \varphi_{1}(x)^{2 m} \leq v_{0}(x) \quad \text { in } \Omega
\end{array}\right.
$$

so that $\underline{v}(x, t) \leq v(x, t)$.

Then $(\underline{u}, \underline{v})$ is a subsolution of (2.1) that blows up at time $T$ defined in (2.4). Then $(u, v)$ blows up at finite time $t_{\mathcal{D}}^{*}$, which is bounded above by $(2.10)$.

\subsection{A lower bound for $t_{\mathcal{D}}^{*}$}

Let $\Omega \subset \mathbb{R}^{3}$ be a bounded domain with the origin inside, star-shaped, and convex in two orthogonal directions, with boundary $\partial \Omega$ smooth enough, and let $[0, \tau], \tau<t_{\mathcal{D}}^{*}$, be the time interval of existence of the solution $(u, v)$ of $(2.1)$.

We define

$$
\Theta(t)=\int_{\Omega} u^{2 p} d x+\int_{\Omega} v^{2 p} d x=\Psi(t)+\Phi(t)
$$

with initial value

$$
\Theta_{0}=\int_{\Omega} u_{0}^{2 p} d x+\int_{\Omega} v_{0}^{2 p} d x
$$

and we prove the following:

Theorem 2.2 Let $\Theta$ be defined in (2.14), and $(u, v)$ be a classical solution of (2.1) that becomes unbounded in the $\Theta$-norm at some finite time $t_{\mathcal{D}}^{*}$. If

$$
p>1, \quad 1<q<2 p,
$$

then

$$
t_{\mathcal{D}}^{*} \geq \begin{cases}\bar{A}^{-1}\left(\frac{1}{2 \Theta_{0}^{2}}\right) & \text { if } 1<q \leq 2, \\ \tilde{A}^{-1}\left(\frac{2}{3 q-2} \frac{1}{\Theta_{0}^{\frac{3}{2} q-1}}\right) & \text { if } 2<q<2 p .\end{cases}
$$

Proof Differentiating (2.14), we have

$$
\Theta^{\prime}=\Psi^{\prime}(t)+\Phi^{\prime}(t),
$$


and using the first equation in (1.1) and the divergence theorem, we obtain

$$
\begin{aligned}
\Psi^{\prime}(t) & =2 p \int_{\Omega} u^{2 p-1} u_{t} d x \\
& =2 p \int_{\Omega} u^{2 p-1} \Delta u d x+2 p k_{1} \int_{\Omega} u^{3 p-1} d x \int_{\Omega} v^{q} d x \\
& =-2 p(2 p-1) \int_{\Omega} u^{2(p-1)}|\nabla u|^{2} d x+2 p k_{1} \int_{\Omega} u^{3 p-1} d x \int_{\Omega} v^{q} d x
\end{aligned}
$$

In order to estimate the last term of (2.19), we use the Hölder inequality, (2.16), and the arithmetic inequality

$$
a^{r} b^{s} \leq r a+s b, \quad r+s=1, a, b>0,
$$

to obtain

$$
\begin{aligned}
& 2 p k_{1} \int_{\Omega} u^{3 p-1} d x \int_{\Omega} v^{q} d x \\
& \quad \leq 2 p k_{1}\left(\int_{\Omega} u^{3 p} d x\right)^{\frac{3 p-1}{3 p}}|\Omega|^{\frac{1}{3 p}} \int_{\Omega} v^{q} d x \\
& \quad \leq \frac{2(3 p-1)}{3} k_{1} \int_{\Omega} u^{3 p} d x+\frac{2|\Omega|}{3} k_{1}\left(\int_{\Omega} v^{q} d x\right)^{3 p} \\
& \quad \leq \frac{2(3 p-1)}{3} k_{1} \int_{\Omega} u^{3 p} d x+\frac{2|\Omega|^{1+3 p-\frac{3}{2} q}}{3} k_{1}\left(\int_{\Omega} v^{2 p} d x\right)^{\frac{3}{2} q} .
\end{aligned}
$$

The term $\int_{\Omega} u^{3 p} d x$ in the last step can be estimated making use of the following Sobolevtype inequality (see Lemma A2 in [19]):

$$
\int_{\Omega} u^{3 p} d x \leq\left\{\frac{3}{2 \rho_{0}} \int_{\Omega} u^{2 p} d x+p\left(1+\frac{d}{\rho_{0}}\right) \int_{\Omega} u^{2 p-1}|\nabla u| d x\right\}^{\frac{3}{2}}
$$

with $\rho_{0}=\min _{\partial \Omega}(x \cdot v)>0$ and $d=\max _{\bar{\Omega}}|x|$, valid in a bounded domain of $\mathbb{R}^{3}$ with the origin inside, star-shaped and convex in two orthogonal directions. By means of (2.22) and the fundamental inequality

$$
(a+b)^{\frac{3}{2}} \leq \sqrt{2}\left(a^{\frac{3}{2}}+b^{\frac{3}{2}}\right) \text { for } a, b>0,
$$

we have

$$
\frac{2(3 p-1)}{3} k_{1} \int_{\Omega} u^{3 p} d x \leq p_{1}\left(\int_{\Omega} u^{2 p} d x\right)^{\frac{3}{2}}+p_{2}\left(\int_{\Omega} u^{2 p-1}|\nabla u| d x\right)^{\frac{3}{2}}
$$

with

$$
p_{1}=2 \sqrt{2} \frac{(3 p-1)}{3} k_{1}\left(\frac{3}{2 \rho_{0}}\right)^{\frac{3}{2}}, \quad p_{2}=2 \sqrt{2} \frac{(3 p-1)}{3} k_{1}\left[p\left(1+\frac{d}{\rho_{0}}\right)\right]^{\frac{3}{2}} .
$$


From the Schwarz inequality and (2.20), in (2.24), we have

$$
\frac{2(3 p-1)}{3} k_{1} \int_{\Omega} u^{3 p} d x \leq p_{1} \Psi^{\frac{3}{2}}+\frac{p_{2}}{4} \epsilon^{3} \Psi^{3}+\frac{3}{4 \epsilon} \int_{\Omega} u^{2(p-1)}|\nabla u| d x
$$

with arbitrary $\epsilon>0$ to be chosen later. Combining (2.25), (2.21), and (2.19), we obtain

$$
\Psi^{\prime}(t) \leq A_{1} \Psi^{\frac{3}{2}}+A_{2} \Psi^{3}+A_{3} \Phi^{\frac{3}{2} q}+A_{4} \int_{\Omega} u^{2(p-1)}|\nabla u|^{2} d x
$$

with

$$
A_{1}=p_{1}, \quad A_{2}=\frac{p_{2}}{4} \epsilon^{3}, \quad A_{3}=\frac{2|\Omega|^{1+3 p-\frac{3}{2} q}}{3} k_{1}, \quad A_{4}=\frac{3}{4 \epsilon}-2 p(2 p-1) .
$$

A similar computation leads to

$$
\Phi^{\prime}(t) \leq A_{1} \Phi^{\frac{3}{2}}+A_{2} \Phi^{3}+A_{3} \Psi^{\frac{3}{2} q}+A_{4} \int_{\Omega} v^{2(p-1)}|\nabla \nu|^{2} d x
$$

Substituting (2.26) and (2.28) into (2.18), we have

$$
\begin{aligned}
\Theta^{\prime}(t) \leq & A_{1}\left(\Psi^{\frac{3}{2}}+\Phi^{\frac{3}{2}}\right)+A_{2}\left(\Psi^{3}+\Phi^{3}\right)+A_{3}\left(\Psi^{\frac{3}{2}} q+\Phi^{\frac{3}{2}} q\right) \\
& +A_{4}\left[\int_{\Omega} u^{2(p-1)}|\nabla u|^{2} d x+\int_{\Omega} v^{2(p-1)}|\nabla v|^{2} d x\right]
\end{aligned}
$$

and using in (2.29) the inequality

$$
a^{c}+b^{c} \leq(a+b)^{c}, \quad c>1, a, b>0
$$

we arrive at

$$
\begin{aligned}
\Theta^{\prime}(t) \leq & A_{1} \Theta^{\frac{3}{2}}+A_{2} \Theta^{3}+A_{3} \Theta^{\frac{3}{2} q} \\
& +A_{4}\left[\int_{\Omega} u^{2(p-1)}|\nabla u|^{2} d x+\int_{\Omega} v^{2(p-1)}|\nabla v|^{2} d x\right] .
\end{aligned}
$$

Now choose $\epsilon$ such that $A_{4}=0$. Then (2.31) becomes

$$
\Theta^{\prime}(t) \leq A_{1} \Theta^{\frac{3}{2}}+A_{2} \Theta^{3}+A_{3} \Theta^{\frac{3}{2} q} .
$$

If $\Theta$ blows up at time $t_{\mathcal{D}}^{*}$, then there exists a time $t_{1} \geq 0$ such that $\Theta(t) \geq \Theta_{0}$ for all $t \geq t_{1}$, and we have

$$
\Theta^{\prime} \leq \begin{cases}A(t) \Theta^{3} & \text { if } 1<q \leq 2, \\ B(t) \Theta^{\frac{3}{2}} q & \text { if } 2<q<2 p\end{cases}
$$

valid for $t \geq t_{1}$ and with

$$
A(t)=A_{1} \Theta_{0}^{-\frac{3}{2}}+A_{2}+A_{3} \Theta_{0}^{\frac{3}{2} q-3}, \quad B(t)=A_{1} \Theta_{0}^{\frac{3}{2}(1-q)}+A_{2} \Theta_{0}^{3\left(1-\frac{1}{2} q\right)}+A_{3} .
$$


Integrating (2.33) from $t_{1}$ to $t_{\mathcal{D}}^{*}$, we obtain the desired lower bound (2.17) with $\bar{A}^{-1}$ and $\tilde{A}^{-1}$ the inverse functions of $\bar{A}(t)=\int_{0}^{t} A(\tau) d \tau$ and $\tilde{A}(t)=\int_{0}^{t} B(\tau) d \tau$, respectively.

\subsection{Nonblow-up case}

In this section, we derive conditions on data such that the blow-up phenomenon cannot occur. Let $(u, v)$ be the solution of (2.1). We consider the auxiliary function $\Theta$ defined in (2.14) and prove the following:

Theorem 2.3 Let $\Omega \subset \mathbb{R}^{3}$ be a bounded domain with the origin inside, star-shaped and convex in two orthogonal directions, with boundary $\partial \Omega$ smooth enough. If (2.16) holds and if

$$
f\left(\Theta_{0}\right)=A_{1} \Theta_{0}^{\frac{1}{2}}+A_{2} \Theta_{0}^{2}+A_{3} \Theta_{0}^{\frac{3}{2} q-1}<C \frac{\lambda_{1}}{p^{2}}, \quad C>0,
$$

with $\lambda_{1}$ the first eigenvalue of the fixed membrane problem (2.6) and $A_{1}, A_{2}, A_{3}$ defined in (2.27), then $\Theta$ cannot blow up.

Proof We follow the proof of Theorem 2.2 up to (2.31), which we rewrite for clarity as

$$
\Theta^{\prime} \leq A_{1} \Theta^{\frac{3}{2}}+A_{2} \Theta^{3}+A_{3} \Theta^{\frac{3}{2} q}+A_{4}\left[\int_{\Omega} u^{2(p-1)}|\nabla u|^{2} d x+\int_{\Omega} v^{2(p-1)}|\nabla v|^{2} d x\right] .
$$

Let us choose $\epsilon$ in the last term of (2.35) such that $A_{4}=-C \leq 0$. Observe that

$$
\left\{\begin{array}{l}
C \int_{\Omega} u^{2(p-1)}|\nabla u|^{2} d x=\frac{C}{p^{2}} \int_{\Omega}\left|\nabla u^{p}\right|^{2} d x, \\
C \int_{\Omega} v^{2(p-1)}|\nabla v|^{2} d x=\frac{C}{p^{2}} \int_{\Omega}\left|\nabla v^{p}\right|^{2} d x .
\end{array}\right.
$$

From the Rayleigh principle we obtain

$$
\begin{aligned}
& \frac{C}{p^{2}}\left[\int_{\Omega}\left|\nabla u^{p}\right|^{2} d x+\int_{\Omega}\left|\nabla v^{p}\right|^{2} d x\right] \\
& \geq \frac{C \lambda_{1}}{p^{2}}\left[\int_{\Omega} u^{2 p} d x+\int_{\Omega} v^{2 p} d x\right]=\frac{C \lambda_{1}}{p^{2}} \Theta .
\end{aligned}
$$

Replacing (2.37) in (2.35), we have

$$
\Theta^{\prime} \leq A_{1} \Theta^{\frac{3}{2}}+A_{2} \Theta^{3}+A_{3} \Theta^{\frac{3}{2} q}-\frac{C \lambda_{1}}{p^{2}} \Theta=-\Theta\left[\frac{C \lambda_{1}}{p^{2}}-f(\Theta)\right]
$$

with $f(\Theta)=A_{1} \Theta^{\frac{1}{2}}+A_{2} \Theta^{2}+A_{3} \Theta^{\frac{3}{2} q-1}$.

If (2.34) and (2.38) hold, then by the comparison principle, $\Theta^{\prime} \leq 0$ for $t>0$, and $\Theta$ cannot blow up. 


\section{Estimates of $t_{\mathcal{N}}^{*}$}

In this section, we consider system (1.1) under the Neumann boundary condition ( $\beta=1$ and $\alpha=0)$ :

$$
\left\{\begin{array}{l}
u_{t}=\Delta u+k_{1}(t) u^{p} \int_{\Omega} v^{q} d x \quad \text { in } \Omega \times\left(0, t_{\mathcal{N}}^{*}\right), \\
v_{t}=\Delta v+k_{2}(t) v^{p} \int_{\Omega} u^{q} d x \quad \text { in } \Omega \times\left(0, t_{\mathcal{N}}^{*}\right), \\
\frac{\partial u}{\partial v}=0, \quad \frac{\partial v}{\partial v}=0 \quad \text { on } \partial \Omega \times\left(0, t_{\mathcal{N}}^{*}\right), \\
u(x, 0)=u_{0}(x) \geq 0, \quad v(x, 0)=v_{0}(x) \geq 0 \quad \text { on } \Omega .
\end{array}\right.
$$

In this case, in order to obtain explicit upper and lower bounds of the blow-up time $t_{\mathcal{N}}^{*}$, we can repeat all the assumptions in Sections 2.1 and 2.2, but now the normal derivative vanishes on the boundary.

\subsection{Upper bound of $t_{\mathcal{N}}^{*}$}

In order to obtain an upper bound of $t_{\mathcal{N}}^{*}$, we seek an unbounded subsolution of problem (3.1):

$$
\left\{\begin{array}{l}
\underline{u}:=s(t)^{n} \phi_{2}(x)^{2 n} \\
\underline{v}:=s(t)^{m} \phi_{2}(x)^{2 m}
\end{array}\right.
$$

with $n, m \in \mathbb{N}$ and $s(t)$ satisfying Lemma 2.1.

Here we put $\gamma, k, a$ as in (2.5) and $a_{1}=2 \mu_{2}$, where $\mu_{2}$ and $\phi_{2}$ are, respectively, the second eigenvalue and eigenfunction of the following free membrane problem:

$$
\left\{\begin{array}{l}
\Delta \phi(x)+\mu \phi(x)=0, \quad x \in \Omega, \\
\frac{\partial \phi(x)}{\partial v}=0, \quad x \in \partial \Omega
\end{array}\right.
$$

with

$$
\int_{\Omega} \phi_{2}^{2}(x) d x=1
$$

Following the steps in Section 2.1, with the above changes, we prove the following:

Theorem 3.1 Let $(u, v)$ be the solution of (3.1). Assume that Lemma 2.1 holds. If

$$
u_{0} \geq s_{0}^{n} \phi_{2}^{2 n}, \quad v_{0} \geq s_{0}^{m} \phi_{2}^{2 m}, \quad n, m \in \mathbb{N},
$$

then $(u, v)$ blows up in finite time $t^{*}$, and

$$
t_{\mathcal{N}}^{*} \leq T=\ln \left[\left(\frac{a s_{0}^{\gamma-1}}{a s_{0}^{\gamma-1}-2 \mu_{2}}\right)^{\frac{1}{2(\gamma-1) \mu_{2}}}\right] .
$$

\subsection{Lower bound of $t_{\mathcal{N}}^{*}$}

Theorem 3.2 Let $\Theta$ be defined in (2.14), and $(u, v)$ be a classical solution of (3.1) that becomes unbounded in the $\Theta$-norm at some finite time $t_{\mathcal{N}}^{*}$. If

$$
p>1, \quad 1<q<2 p,
$$


then

$$
t_{\mathcal{N}}^{*} \geq \begin{cases}\bar{B}^{-1}\left(\frac{1}{2 \Theta_{0}^{2}}\right) & \text { if } 1<q \leq 2 \\ \tilde{B}^{-1}\left(\frac{2}{3 q-2} \frac{1}{\Theta_{0}^{\frac{3}{2} q-1}}\right) & \text { if } 2<q<2 p\end{cases}
$$

The proof follows the reasoning in Section 2.2: taking into account that when we apply the divergence theorem in (2.19), the Neumann boundary condition must be used, we get (2.21). Now we remark that the Sobolev inequality (2.22) also holds for a function with vanishing normal derivative on the boundary. In this way, the first-order differential inequality (2.33) is obtained from which we achieve (3.7).

\subsection{Nonblow-up case}

Regarding the nonblow-up case under the Neumann boundary condition, we cannot use the Rayleigh principle. We now prove a lemma that plays an important role in the proof of Theorem 3.3.

Lemma 3.1 Let $\Omega$ be a convex domain in $\mathbb{R}^{3}$ with sufficiently smooth boundary. If $w$ is a $C^{1}$-function, then

$$
\int_{\Omega}\left|\nabla w^{\frac{n}{2}}\right|^{2} d x \geq m_{1}\left(\int_{\Omega} w^{\frac{3 n}{2}} d x\right)^{\frac{2}{3}}-m_{2} \int_{\Omega} w^{n} d x
$$

with $m_{1}$, and $m_{2}$ defined further.

Proof We recall inequality (2.16) in [20]:

$$
\int_{\Omega} w^{\frac{3 n}{2}} d x \leq \frac{1}{3^{\frac{3}{4}}}\left\{\frac{3}{2 \rho_{0}} \int_{\Omega} w^{n} d x+\left(1+\frac{d}{\rho_{0}}\right)\left(\int_{\Omega} w^{n} d x\right)^{\frac{1}{2}}\left(\int_{\Omega}\left|\nabla w^{\frac{n}{2}}\right|^{2} d x\right)^{\frac{1}{2}}\right\}^{\frac{3}{2}}
$$

valid in a convex domain $\Omega \in \mathbb{R}^{3}$ with sufficiently smooth boundary and with $\rho_{0}=$ $\min _{\partial \Omega}(x \cdot v)>0$ and $d=\max _{\bar{\Omega}}|x|$.

Using the arithmetic inequality (2.20) in (3.9), we have

$$
\left(\int_{\Omega} w^{\frac{3 n}{2}} d x\right)^{\frac{2}{3}} \leq c_{1} \int_{\Omega} w^{n} d x+c_{2} \int_{\Omega}\left|\nabla w^{\frac{n}{2}}\right|^{2} d x
$$

with $c_{1}=\frac{\sqrt{3}}{2 \rho_{0}}+\frac{\epsilon_{1}}{2 \sqrt{3}}, c_{2}=\frac{1}{2 \sqrt{3} \epsilon_{1}}, \epsilon_{1}>0$.

Thus, by (3.10) we can take (3.8) with $m_{1}=2 \sqrt{3} \epsilon_{1}$ and $m_{2}=3 \frac{\epsilon_{1}}{\rho_{0}}+\epsilon_{1}^{2}$.

Now, we can prove following theorem.

Theorem 3.3 Let $\Omega \subset \mathbb{R}^{3}$ be a bounded domain with the origin inside, star-shaped and convex in two orthogonal directions, with boundary $\partial \Omega$ smooth enough. We assume that

$$
m_{1}|\Omega|^{-\frac{1}{3}}-m_{2} \geq 0
$$

with $m_{1}, m_{2}$ defined in Lemma 3.1. 
If (2.16) holds and if

$$
f\left(\Theta_{0}\right)=A_{1} \Theta_{0}^{\frac{1}{2}}+A_{2} \Theta_{0}^{2}+A_{3} \Theta_{0}^{\frac{3}{2} q-1}<\bar{C}, \quad \bar{C}>0,
$$

with $A_{1}, A_{2}, A_{3}$ defined in (2.27), then $\Theta$ cannot blow up.

Proof Following the proof of Theorem 2.3 up to (2.36), we have

$$
\Theta^{\prime} \leq A_{1} \Theta^{\frac{3}{2}}+A_{2} \Theta^{3}+A_{3} \Theta^{\frac{3}{2} q}-\frac{C}{p^{2}}\left[\int_{\Omega}\left|\nabla u^{p}\right|^{2} d x+\int_{\Omega}\left|\nabla \nu^{p}\right|^{2} d x\right] .
$$

In the last term of (3.13), now using (3.8) with $w=u$ or $w=v$ and $n=2 p$, we obtain

$$
\begin{aligned}
& \frac{C}{p^{2}}\left[\int_{\Omega}\left|\nabla u^{p}\right|^{2} d x+\int_{\Omega}\left|\nabla v^{p}\right|^{2} d x\right] \\
& \quad \geq m_{1}\left[\left(\int_{\Omega} u^{3 p} d x\right)^{\frac{2}{3}}+\left(\int_{\Omega} v^{3 p} d x\right)^{\frac{2}{3}}\right]-\frac{C}{p^{2}} m_{2} \Theta .
\end{aligned}
$$

By the Hölder inequality we can deduce

$$
\left(\int_{\Omega} u^{3 p} d x\right)^{\frac{2}{3}} \geq|\Omega|^{-\frac{1}{3}} \int_{\Omega} u^{2 p} d x, \quad\left(\int_{\Omega} v^{3 p} d x\right)^{\frac{2}{3}} \geq|\Omega|^{-\frac{1}{3}} \int_{\Omega} v^{2 p} d x
$$

Replacing (3.15) in (3.14), we obtain

$$
\begin{aligned}
& \frac{C}{p^{2}}\left[\int_{\Omega}\left|\nabla u^{p}\right|^{2} d x+\int_{\Omega}\left|\nabla v^{p}\right|^{2} d x\right] \\
& \geq \frac{C}{p^{2}}\left(m_{1}|\Omega|^{-\frac{1}{3}}-m_{2}\right) \Theta .
\end{aligned}
$$

Substituting (3.16) into (3.13), we arrive at

$$
\Theta^{\prime} \leq-\frac{C}{p^{2}}\left(m_{1}|\Omega|^{-\frac{1}{3}}-m_{2}\right) \Theta+A_{1} \Theta^{\frac{3}{2}}+A_{2} \Theta^{3}+A_{3} \Theta^{\frac{3}{2} q} .
$$

In view of (3.11), (3.17) can be rewritten as

$$
\Theta^{\prime} \leq-\Theta[\bar{C}-f(\Theta)]
$$

with $f(\Theta)=A_{1} \Theta^{\frac{1}{2}}+A_{2} \Theta^{2}+A_{3} \Theta^{\frac{3}{2} q-1}$ and $\bar{C}=\frac{C}{p^{2}}\left(m_{1}|\Omega|^{-\frac{1}{3}}-m_{2}\right)$.

If (3.12) and (3.18) hold, then, by the comparison principle, $\Theta^{\prime} \leq 0$ for $t>0$, and $\Theta$ cannot blow up.

\section{Estimates of $t_{\mathcal{R}}^{*}$}

In the case of Robin boundary condition, the extension of Theorems 2.1, 2.2, and 2.3 is not so immediate. 
We consider problem (1.1) with $\beta=1, \alpha>0$ :

$$
\left\{\begin{array}{l}
u_{t}=\Delta u+k_{1}(t) u^{p} \int_{\Omega} v^{q} d x \quad \text { in } \Omega \times\left(0, t_{\mathcal{R}}^{*}\right), \\
v_{t}=\Delta v+k_{2}(t) v^{p} \int_{\Omega} u^{q} d x \quad \text { in } \Omega \times\left(0, t_{\mathcal{R}}^{*}\right), \\
\frac{\partial u}{\partial v}+\alpha u=0 \quad \text { on } \partial \Omega \times\left(0, t_{\mathcal{R}}^{*}\right), \\
\frac{\partial v}{\partial v}+\alpha v=0 \quad \text { on } \partial \Omega \times\left(0, t_{\mathcal{R}}^{*}\right), \\
u(x, 0)=u_{0}(x) \quad \text { on } \Omega, \\
v(x, 0)=v_{0}(x) \quad \text { on } \Omega .
\end{array}\right.
$$

\subsection{Upper bound of $t_{\mathcal{R}}^{*}$}

We look for a blowing up subsolution of problem (4.1):

$$
\left\{\begin{array}{l}
\underline{u}:=s(t)^{n} \psi_{1}(x)^{2 n} \\
\underline{v}:=s(t)^{m} \psi_{1}(x)^{2 m}
\end{array}\right.
$$

with $n, m \in \mathbb{N}$ and $s(t)$ satisfying Lemma 2.1.

Here we put $\gamma, k, a$ as in (2.5) and $a_{1}=2 \xi_{1}$, where $\xi_{1}$ and $\psi_{1}$ are, respectively, the first eigenvalue and the corresponding eigenfunction of the elastically supported membrane problem

$$
\left\{\begin{array}{l}
\Delta \psi(x)+\xi \psi(x)=0, \quad \psi(x)>0, x \in \Omega, \\
\frac{\partial \psi(x)}{\partial \nu}+\alpha \psi=0, \quad x \in \partial \Omega
\end{array}\right.
$$

with

$$
\int_{\Omega} \psi_{1}^{2}(x) d x=1
$$

Following the steps in Section 2.1, with the right changes, the following result holds.

Theorem 4.1 Let $(u, v)$ be the solution of (4.1). Assume that Lemma 2.1 holds. If

$$
u_{0} \geq s_{0}^{n} \psi_{2}^{2 n}, \quad v_{0} \geq s_{0}^{m} \psi_{2}^{2 m}, \quad n, m \in \mathbb{N},
$$

then $(u, v)$ blows up in finite time $t^{*}$, and

$$
t_{\mathcal{R}}^{*} \leq T=\ln \left[\left(\frac{a s_{0}^{\gamma-1}}{a s_{0}^{\gamma-1}-2 \xi_{1}}\right)^{\frac{1}{2(\gamma-1) \xi_{1}}}\right] .
$$

\subsection{Lower bound of $t_{\mathcal{R}}^{*}$}

In order to obtain an explicit lower bound of $t_{\mathcal{R}}^{*}$ of the solution of problem (4.1), we consider the auxiliary function (2.14), and we follow the arguments in Section 2.2 to prove the following: 
Theorem 4.2 Let $\Theta$ be defined in (2.14), and $(u, v)$ be a classical solution of (4.1) that becomes unbounded in the $\Theta$-norm at some finite time $t_{\mathcal{R}}^{*}$. If

$$
p>1, \quad 1<q<2 p,
$$

then

$$
t_{\mathcal{R}}^{*} \geq \begin{cases}\bar{B}^{-1}\left(\frac{1}{2 \Theta_{0}^{2}}\right) & \text { if } 1<q \leq 2, \\ \tilde{B}^{-1}\left(\frac{2}{3 q-2} \frac{1}{\Theta_{0}^{\frac{3}{2} q-1}}\right) & \text { if } 2<q<2 p .\end{cases}
$$

Proof Differentiating (2.14), we have

$$
\Theta^{\prime}=\Psi^{\prime}(t)+\Phi^{\prime}(t)
$$

and using the first equation in (4.1) and the divergence theorem, we obtain

$$
\begin{aligned}
\Psi^{\prime}(t)= & 2 p \int_{\Omega} u^{2 p-1} u_{t} d x=2 p \int_{\Omega} u^{2 p-1} \Delta u d x+2 p k_{1} \int_{\Omega} u^{3 p-1} d x \int_{\Omega} v^{q} d x \\
= & -2 p \alpha \int_{\partial \Omega} u^{2 p} d s-2 p(2 p-1) \int_{\Omega} u^{2(p-1)}|\nabla u|^{2} d x \\
& +2 p k_{1} \int_{\Omega} u^{3 p-1} d x \int_{\Omega} v^{q} d x .
\end{aligned}
$$

In order to estimate the last term of (4.9), following the steps in the proof of Theorem 2.2, we obtain

$$
\begin{aligned}
& 2 p k_{1} \int_{\Omega} u^{3 p-1} d x \int_{\Omega} v^{q} d x \\
& \quad \leq 2 p k_{1}\left(\int_{\Omega} u^{3 p} d x\right)^{\frac{3 p-1}{3 p}}|\Omega|^{\frac{1}{3 p}} \int_{\Omega} v^{q} d x \\
& \quad \leq \frac{2(3 p-1)}{3} k_{1} \int_{\Omega} u^{3 p} d x+\frac{2|\Omega|^{1+3 p-\frac{3}{2} q}}{3} k_{1}\left(\int_{\Omega} v^{2 p} d x\right)^{\frac{3}{2} q} \\
& \quad \leq A_{1} \Psi^{\frac{3}{2}}+A_{2} \Psi^{3}+A_{3} \Phi^{\frac{3}{2} q}
\end{aligned}
$$

with $A_{1}, A_{2}, A_{3}$ defined in (2.27).

Now we estimate the first term in (4.9). To this end, we use the variational definition of the first eigenvalue $\xi_{1}$ of problem (4.3). We have

$$
\begin{aligned}
-2 p \alpha \int_{\partial \Omega} u^{2 p} d s & \leq 2 p(2 p-1) \int_{\Omega} u^{2(p-1)}|\nabla u|^{2} d x-2 p \xi_{1} \int_{\Omega} u^{2 p} d x \\
& =2 p(2 p-1) \int_{\Omega} u^{2(p-1)}|\nabla u|^{2} d x-2 p \xi_{1} \Psi .
\end{aligned}
$$

Replacing (4.10) and (4.11) in (4.9), we have

$$
\Psi^{\prime}(t) \leq A_{1} \Psi^{\frac{3}{2}}+A_{2} \Psi^{3}+A_{3} \Phi^{\frac{3}{2} q}-2 p \xi_{1} \Psi .
$$


Similarly, for $\Phi$, we have

$$
\Phi^{\prime}(t) \leq A_{1} \Phi^{\frac{3}{2}}+A_{2} \Phi^{3}+A_{3} \Psi^{\frac{3}{2} q}-2 p \xi_{1} \Phi
$$

Substituting (4.12) and (4.13) into (4.8), we obtain

$$
\begin{aligned}
\Theta^{\prime}(t) & \leq A_{1}\left(\Psi^{\frac{3}{2}}+\Phi^{\frac{3}{2}}\right)+A_{2}\left(\Psi^{3}+\Phi^{3}\right)+A_{3}\left(\Psi^{\frac{3}{2} q}+\Phi^{\frac{3}{2} q}\right)-2 p \xi_{1} \Theta \\
& \leq A_{1} \Theta^{\frac{3}{2}}+A_{2} \Theta^{3}+A_{3} \Theta^{\frac{3}{2} q}+2 p \xi_{1} \Theta
\end{aligned}
$$

where in the last step we have used the inequality

$$
a^{c}+b^{c} \leq(a+b)^{c}, \quad c>1, a, b>0 .
$$

If $\Theta$ blows up at time $t_{\mathcal{R}}^{*}$, then there exists a time $t_{1} \geq 0$ such that $\Theta(t) \geq \Theta_{0}$ for $t \geq t_{1}$, and we have

$$
\Theta^{\prime} \leq \begin{cases}A(t) \Theta^{3} & \text { if } 1<q \leq 2, \\ B(t) \Theta^{\frac{3}{2}} q & \text { if } 2<q<2 p\end{cases}
$$

valid for $t \geq t_{1}$ and with

$$
\begin{aligned}
& A(t)=A_{1} \Theta_{0}^{-\frac{3}{2}}+A_{2}+A_{3} \Theta_{0}^{\frac{3}{2} q-3}+2 p \xi_{1} \Theta_{0}^{-2}, \\
& B(t)=A_{1} \Theta_{0}^{\frac{3}{2}(1-q)}+A_{2} \Theta_{0}^{3\left(1-\frac{1}{2} q\right)}+A_{3}+2 p \xi_{1} \Theta_{0}^{1-\frac{3}{2} q} .
\end{aligned}
$$

Integrating (4.15) from $t_{1}$ to $t_{\mathcal{R}}^{*}$, we obtain the desired lower bound (4.7) with $\bar{B}^{-1}$ and $\tilde{B}^{-1}$ the inverse functions of $\bar{B}(t)=\int_{0}^{t} A(\tau) d \tau$ and $\tilde{B}(t)=\int_{0}^{t} B(\tau) d \tau$, respectively.

\subsection{Nonblow-up case}

Theorem 4.3 Let $\Omega \subset \mathbb{R}^{3}$ be a bounded domain with the origin inside, star-shaped and convex in two orthogonal directions, with boundary $\partial \Omega$ smooth enough. If (2.16) holds and if

$$
f\left(\Theta_{0}\right)=A_{1} \Theta_{0}^{\frac{1}{2}}+A_{2} \Theta_{0}^{2}+A_{3} \Theta_{0}^{\frac{3}{2} q-1}<2 p \xi_{1}
$$

with $A_{1}, A_{2}, A_{3}$ defined in (2.27) and $\xi_{1}$ the first eigenvalue of (4.3), then $\Theta$ cannot blow up. Proof We follow the proof of Theorem 4.2 up to (4.14). We have

$$
\Theta^{\prime}(t) \leq-\Theta\left[2 p \xi_{1}-f(\Theta)\right]
$$

with $f(\Theta)=A_{1} \Theta^{\frac{1}{2}}+A_{2} \Theta^{2}+A_{3} \Theta^{\frac{3}{2} q-1}$.

If (4.16) and (4.17) hold, then by the comparison principle, $\Theta^{\prime} \leq 0$ for $t>0$, and $\Theta$ cannot blow up. 
Authors' contributions

Both authors contributed equally and significantly in writing this paper. Both authors read and approved the final manuscript.

\section{Acknowledgements}

The authors are very grateful to the anonymous referee for his/her helpful suggestions and comments.

The authors are members of G.N.A.M.P.A. (I.N.d.A.M.) and were supported by University of Cagliari.

Received: 10 September 2016 Accepted: 29 November 2016 Published online: 03 January 2017

\section{References}

1. Sperb, R: Maximum Principles and Their Applications. Math. in Sci. and Engineering, vol. 157. Academic Press, New York (1981)

2. Day, WA: A decreasing property of solutions of parabolic equations with applications to thermoelasticity. Q. Appl. Math. 40(4), 468-475 (1983)

3. Day, WA: Heat Conduction Within Linear Thermoelasticity. Springer Tracts in Natural Philosophy, vol. 30. Springer, New York (1985)

4. Friedman, A: Monotonic decay of solutions of parabolic equations with nonlocal boundary conditions. Q. Appl. Math. 44(3), 401-407 (1986)

5. Pao, CV: Asymptotic behavior of solutions of reaction-diffusion equations with nonlocal boundary conditions. J. Comput. Appl. Math. 88(1), 225-238 (1998)

6. Kong, LH, Wang, MX: Global existence and blow-up of solutions to a parabolic system with nonlocal sources and boundaries. Sci. China Ser. A 50(9), 1251-1266 (2007)

7. Souplet, P: Uniform blow-up profiles and boundary behavior for diffusion equations with nonlocal nonlinear source. J. Differ. Equ. 153(2), 374-406 (1999)

8. Zheng, S, Kong, L: Roles of weight functions in a nonlinear nonlocal parabolic system. Nonlinear Anal. 68(8), 2406-2416 (2008)

9. Souplet, P: Blow-up in nonlocal reaction-diffusion equations. SIAM J. Math. Anal. 29, 1301-1334 (1998)

10. Marras, M, Vernier Piro, S: Reaction-diffusion problems under non-local boundary conditions with blow-up solutions. J. Inequal. Appl. 2014, 167 (2014)

11. Marras, M, Vernier Piro, S: Explicit estimates for blow-up solutions to parabolic systems under nonlocal boundary conditions. Comptes Rendus de L'Académie Bulgare des Sciences 67(4), 459-466 (2014)

12. Marras, M, Vernier-Piro, S, Viglialoro, G: Blow-up phenomena in chemotaxis systems with a source term. Mathematical Methods in the Applied Sciences. doi:10.1002/mma.3728

13. Viglialoro, G: Blow-up time of a Keller-Segel-type system with Neumann and Robin boundary conditions. Differ. Integral Equ. 29(3-4), 359-376 (2016)

14. Chen, S: Global existence and blowup for quasilinear parabolic equations not in divergence form. J. Math. Anal. Appl. 401(1), 298-306 (2013)

15. Pao, CV: Blowing-up of solution for a nonlocal reaction-diffusion problem in combustion theory. J. Math. Anal. Appl. $166,591-600$ (1992)

16. Henrot, A: Extremum Problems for Eigenvalues of Elliptic Operators. Birkhäuser, Basel (2006)

17. Lubarda, VA, Marzani, A: Viscoelastic response of thin membranes with application to red blood cells. Acta Mechanica 202(116) (2009). doi:10.1007/s00707-008-0005-y

18. Ruggieri, M, Valenti, A: Exact solutions for a nonlinear model of dissipative media. J. Math. Phys. 52(4), 043520 (2011). doi:10.1063/1.3577958

19. Payne, LE, Philippin, GA, Vernier Piro, S: Blow up phenomena for a semilinear heat equation with nonlinear boundary condition, II. Nonlinear Anal. 73, 971-978 (2010)

20. Payne, LE, Schaefer, PW: Lower bounds for blow-up time in parabolic problems under Neumann conditions. Appl. Anal. 85, 1301-1311 (2006)

\section{Submit your manuscript to a SpringerOpen ${ }^{\circ}$ journal and benefit from:}

- Convenient online submission

- Rigorous peer review

- Immediate publication on acceptance

- Open access: articles freely available online

- High visibility within the field

- Retaining the copyright to your article 